\title{
Threshold Pion Photo- and Electroproduction in Chiral Perturbation Theory
}

\author{
Véronique Bernard \\ Centre de Recherches Nucléaires et Université Louis Pasteur de Strasbourg, \\ Physique Théorique, Bat. 40, BP28, 67037 Strasbourg Cedex, France
}

\section{Introduction}

Threshold pion photo- and electroproduction off nucleons allow us, by the use of a well-understood probe, to test our understanding of the strong interaction at low energies, i.e. in the non-perturbative regime. Over the last few years, renewed interest in these reactions has emerged. This was first triggered through precise new data on neutral pion photoproduction [1] which lead to a controversy about their theoretical interpretations. Furthermore, new data on $\pi^{0}$ electroproduction [2] being obtained with an unprecedented accuracy have given further constraints on the understanding of these fundamental processes in the non-perturbative regime of QCD. While the study of these reactions, also with charged pions in the final state, continues on the experimental as well as on the theoretical side, complementary information can be gained from the two-pion production process $\gamma N \rightarrow \pi \pi N$, where $\mathrm{N}$ denotes the nucleon and $\gamma$ the real or virtual photon. Presently available data on this reaction focus on the resonance region (excitation energies close to the first strong resonance excitation of the nucleon, the $\Delta(1232)$ ) and above, however, an attempt to measure the $\pi^{0} \pi^{0} p$ channel very close to threshold is actually being made in Mainz [3].

I will report here on a systematic analysis of the processes $\gamma^{(\star)} N \rightarrow \pi N$ and $\gamma N \rightarrow$ $\pi \pi N$ in the threshold region making use of baryon chiral perturbation theory (CHPT). Other aspects of nucleon chiral perturbation theory are discussed in these proceedings by Ulf-G. Meißner. In general, CHPT allows one to systematically investigate the strictures of the spontaneously broken chiral symmetry of QCD. It is based on the observation that in the three flavor sector of QCD, the quark masses are small and that the theory in the limit of vanishing quark masses admits an exact chiral symmetry. The latter is dynamically broken which leads to the appearance of massless pseudoscalar excitations, the Goldstone bosons. In the real word, the quark masses are not exactly zero and thus the Goldstone bosons acquire a small mass. The interaction of these particles with each other and matter fields like e.g. the nucleons are weak at low energies as mandated by Goldstone's theorem. This fact is at the heart of CHPT which amounts to a systematic and simultaneous expansion of the QCD Green functions in small momenta and quark masses. To perturbatively restore unitarity it is mandatory to consider pion loop diagrams. Here we will work in the one-loop approximation which has been shown to be of sufficient accuracy for many threshold phenomena. For a review see H. Leutwyler [4] and Ulf-G. Meißner [5].

Talk given at the workshop on Chiral Dynamics: Theory and Experiments, MIT, Cambridge, USA, July 25 - July 29, 1994. 


\section{Effective Lagrangian}

In this section I will be rather short. For more details see A. Manohar and UlfG. Meißner, these proceedings. To systematically work out the consequences of the spontaneously broken chiral symmetry at low energies, one makes use of an effective Lagrangian of the asymptotically observed fields, in our case the Goldstone bosons (pions) and the nucleons (i.e. proton and neutron). We work in flavor $\mathrm{SU}(2)$ and mostly in the isospin limit $m_{u}=m_{d}=\hat{m}$ and consider terms up to and including $\mathcal{O}\left(q^{4}\right)$ where $q$ denotes a generic small momentum, in the heavy mass formulation. This formulation has the advantage that there is a one-to-one correspondence between loops and powers of $q$. Indeed, this is not the case in the relativistic calculation where things are complicated by the fact that the nucleon mass does not vanish in the chiral limit. The effective Lagrangian can be written as:

$$
\mathcal{L}_{\text {eff }}=\mathcal{L}_{\pi N}^{(1)}+\mathcal{L}_{\pi N}^{(2)}+\mathcal{L}_{\pi N}^{(3)}+\mathcal{L}_{\pi N}^{(4)}+\mathcal{L}_{\pi \pi}^{(2,4)}
$$

where $\mathcal{L}_{\pi N}^{(1)}$ represents the non-linear $\sigma$ model coupled to nucleons. Four parameters enter the theory to lowest order, namely the pion decay constant $F$, the axial coupling constant $\stackrel{\circ}{g}_{A}$, the nucleon mass $\stackrel{\circ}{m}$ ( $\stackrel{\circ}{m}$ only enters implicitely through the non-relativistic energy-momentum relation $p=\stackrel{m}{m}$. It does not appear in the Lagrangian to lowest order) and the leading term in the quark mass expansion of the pion mass $M_{\pi} . F, \stackrel{\circ}{g}_{A}$ and $\stackrel{\circ}{m}$ denotes quantities in the chiral limit, thus differing from the physical ones through terms proportional to the current quark mass. The vertices from $\mathcal{L}_{\pi N}^{(1)}$ needed to perform a one-loop calculation of photo- and electroproduction are the pseudovector $\pi N N$ vertex and the well-known Weinberg and Kroll Ruderman terms. Notice that working in the Coulomb gauge $\epsilon_{0}=0$ (as we will do in the following) there is no direct coupling of the photon to the nucleon (proportional to $\epsilon \cdot v)$. This of course simplifies the calculation since many diagrams vanish. $\mathcal{L}_{\pi N}^{(2,3,4)}$ contain $1 / m$ corrections and counterterms. The a priori unknown coefficients of these counterterms are the so-called low energy constants. For a detailed discussion of their estimates see G. Ecker, these proceedings. I will come back to the ones entering the particular reactions discussed here in section 3.1. Note that $\stackrel{\circ}{\kappa}_{S, V}$, the isoscalar and isovector anomalous magnetic moments of the nucleon in the chiral limit, enter $\mathcal{L}_{\pi N}^{(2)}$ as such low energy constants. Let me finally point out that another simplification arises in the heavy mass formulation from the fact that the algebra only involves the velocity operator $v$ and the covariant spin operator $S$. For more details, see for example ref.[6].

\section{Threshold Pion Photoproduction}

Let us consider the reaction $\gamma(k)+N_{1}\left(p_{1}\right) \rightarrow \pi^{a}(q)+N_{2}\left(p_{2}\right)$ with $N_{1,2}$ denoting protons and/or neutrons and ' $a$ ' refers to the charge of the produced pion. In the case of real photons $\left(k^{2}=0\right)$ one talks of photoproduction whereas for virtual photons (radiated off an electron beam) the process is called electroproduction. Of particular interest is the threshold region where the photon has just enough energy to produce the pion at rest or with a very small three-momentum. In this kinematical regime, 
it is advantageous to perform a multipole decomposition since at threshold only the $S$-waves survive. These multipoles are labelled $E, M, L_{l \pm}$, where $E, M, L$ stands for electric, magnetic, longitudinal respectively ( $L$ of course does not appear in photoproduction where the photons are transverse), $l=0,1,2, \ldots$ the pion orbital angular momentum and the \pm refers to the total angular momentum of the pion-nucleon system, $j=l \pm 1 / 2$. They parametrize the structure of the nucleon as probed with low energy photons.

\subsection{CHPT calculations}

Let us first concentrate on the photoproduction case. We will consider here only the neutral channel. A detailed discussion of the charged ones in the relativistic formalism is given in Ref.[7] and the experimental status is reported by J. Bergstrom and M. Kovash (these proceedings). The $\mathrm{T}$-matrix depends on four amplitudes and takes the following form close to threshold where it is legitimate to keep only the $S$ - and $P$-waves:

$$
T \cdot \vec{\epsilon}=\vec{\sigma} \cdot \vec{\epsilon}\left(E_{0+}+\hat{k} \cdot \hat{q} P_{1}\right)+\vec{\sigma} \cdot \hat{k} \vec{\epsilon} \cdot \hat{q} P_{2}+(\hat{q} \times \hat{k}) \cdot \vec{\epsilon} P_{3}
$$

The quantities $P_{1,2,3}$ represent the following combinations of the three $P$-waves, $E_{1+}, M_{1+}$ and $M_{1-}$,

$$
\begin{aligned}
& P_{1}=3 E_{1+}+M_{1+}-M_{1-} \\
& P_{2}=3 E_{1+}-M_{1+}+M_{1-} \\
& P_{3}=2 M_{1+}+M_{1-}
\end{aligned}
$$

These four amplitudes are easily calculable within CHPT. Let us first discuss the $\mathcal{O}\left(q^{3}\right)$ results. I should point out that since $\epsilon$, the polarization vector of the photon, is a quantity of order $q$ in the chiral counting (it enters the covariant derivative at the same level as $\left.\partial_{\mu}\right) E_{0+}$ and the $P_{i}$ are therefore quantities of order $q^{2}$.

- At tree level one has contributions from $\mathcal{L}_{\pi N}^{(2,3)}$ which bring corrections of order $E / m^{2}$ and $E^{2} / m^{3}$, respectively, where $E$ is a generic small momentum which in the present case can either be $\omega$, the $\pi^{0}$ center of mass energy, $|\vec{q}|$ its three momentum (for the $P$-waves) and $M_{\pi}$.

- Loops start at this order. The well-known rescattering graph and the very important, as we will see later, triangle graph contribute to $E_{0+}$ while, as shown in Fig.1 two other graphs contribute to the $P$-waves. It turns out that these loop contributions are finite, of the type $E^{2} / F_{\pi}^{3}$ and cancel for $P_{3}$. One of course should add the diagrams which contribute to mass and coupling constant renormalization. We don't show them here.

- Three counterterms appear at $\mathcal{O}\left(q^{3}\right)$. One, $\kappa_{p}$, the anomalous magnetic moment of the proton in the chiral limit, enters $\mathcal{L}_{\pi N}^{(2)}$ as discussed in the previous section. Note that in contrast to the relativistic result [8] $\kappa_{p}$ is almost entirely given by the counterterm. It contributes to all the multipoles except $P_{3}$ at variance with the two other counterterms which only contribute to $P_{3}$. These, $b_{22}$ and $b_{14}$ which come from $\mathcal{L}_{\pi N}^{(2)}$ and $\mathcal{L}_{\pi N}^{(3)}$ respectively, have been first written down by Ecker [9]. They are infinite but they enter through the combination $b=4 b_{14}+g_{A} b_{22}$ which just cancels these infinities. Effectively there is thus only one counterterm $b$ entering $P_{3}$. There are two ways of determining these counterterms (both will be 
investigated here). The first one which is in the spirit of CHPT, is to fit them to the total and differential photocrossection. One then predicts the multipoles and all the electroproduction observables. An alternative way is to use the principle of resonance saturation demonstrated by Ecker et al. [10] in the meson sector. The low energy constants are evaluated through the exchange of resonances (in our case the $\Delta, \rho$ and $\omega)$. This is often called the QCD version of Vector Meson Dominance. This introduces of course some scale dependence since the couterterms are obtained at a certain scale which is given by the resonance masses. For more detail, see G. Ecker, these proceedings.

Fig.1 One-loop diagrams contributing to $E_{0+}$ (two first) and $P_{i}$ 's (two last).

I will not enter into the discussion of the $\mathcal{O}\left(q^{4}\right)$ terms in detail here. Let me just say that the loops are calculated with one vertex from $\mathcal{L}_{\pi N}^{(2)}$ and one from $\mathcal{L}_{\pi N}^{(1)}$, or with vertices from $\mathcal{L}_{\pi N}^{(1)}$ but a propagator from $\mathcal{L}_{\pi N}^{(2)}$ or are $1 / m$ corrections to the loops appearing at $\mathcal{O}\left(q^{3}\right)$. It turns out that there are two more counterterms from $\mathcal{L}_{\pi N}^{(4)}$ contributing to $E_{0+}$ at this next order. Thus, the general expressions for $E_{0+}$ and the $P$-waves to $\mathcal{O}\left(q^{4}\right)$ and $\mathcal{O}\left(q^{3}\right)$, respectively, are:

$$
\begin{aligned}
E_{0+} & =\frac{\alpha}{m^{2}} E+\left(\frac{\beta}{m^{3}}+\frac{\gamma}{F^{3}}+\kappa_{p} \delta\right) E^{2}+\left(\frac{\epsilon}{m^{4}}+\frac{\mu}{m F^{3}}\right) E^{3}+d_{5} M_{\pi}^{2} \omega+d_{6} \omega^{3} \\
P_{1,2} & =\frac{\alpha^{\prime}}{m^{2}}|\vec{q}|+\left(\frac{\beta^{\prime}}{m^{3}}+\frac{\gamma^{\prime}}{F^{3}}+\kappa_{p} \delta^{\prime}\right)|\vec{q}| E \\
P_{3} & =\text { small Born }+d_{7}|\vec{q}| \omega
\end{aligned}
$$

where $d_{i}$ are quantities proportional to the low energy constants, $\alpha, \beta, \delta, \epsilon$ coming from tree diagrams are real functions of $\omega$ and $\gamma$ and $\mu$ which are loop contributions are complex function of $\omega$ for $\omega>M_{\pi^{+}}$(same holds for the ' quantities), thus restoring unitarity in a perturbative manner. An important fact is that to lowest order $E_{0+}$ and the $P$ 's have the same chiral power. Indeed, $P_{1,2}$ are proportional to $|\vec{q}|$ and not $|\vec{q}||\vec{k}|$ as it is usually assumed. However since $|\vec{k}|$ is a very slowly varying function close to threshold it turns out that this approximation is rather good. Note that apart from a small Born term $P_{3}$ is essentially given by a counterterm. 


\subsection{Low energy theorems}

A low energy theorem was derived in the seventies [11], giving $E_{0+}$ for neutral pion photoproduction at threshold as a series in $\mu=M_{\pi} / m=1 / 7$ up to and including $\mathcal{O}\left(\mu^{2}\right)$ in terms of physical quantities only. This "LET" was based on chiral symmetry and on some so-called harmless assumptions. It turned out that it was in contradiction with the latest experimental informations [1]. The theorem has been therefore reconsidered by several authors [12] (and confirmed by some) and the interpretation of the experimental data has been critically examined $[13,14]$. Before entering the discussion of this LET, let me first emphasize that CHPT is not a model but is a method for solving QCD at low energy. As it is well-known and most clearly spelled out by Weinberg [15] CHPT embodies the very general principle of gauge invariance, analyticity, crossing and PCAC (pion pole dominance). Therefore to lowest order, one recovers the venerable current algebra LETs, which are based on these principles. The effective chiral lagrangian is simply a tool to calculate these but also all next-toleading order corrections in a systematic and controlled fashion. Thus any theory or model which claims to embody PCAC should lead to the same result as CHPT if one goes to the same level of sophistication. Performing a one-loop CHPT calculation, $E_{0+}$ at threshold is given by:

$$
E_{0+}=-\frac{e g_{\pi N}}{8 \pi m} \mu\left\{1-\left[\frac{1}{2}\left(3+\kappa_{p}\right)+\left(\frac{m}{4 F_{\pi}}\right)^{2}\right] \mu+\mathcal{O}\left(\mu^{2}\right)\right\},
$$

with $\kappa_{p}=3.71$ the anomalous magnetic moment of the proton. The second term in the square brackets was not appearing in the original "LET". It is the new one found in the CHPT calculation. It stems from the so-called triangle diagram and its crossed partner. If one expresses $E_{0+}$ to lowest order in terms of the conventional Lorentz invariant function $A_{1}, E_{0+}=\mu A_{1}$, one finds that these diagrams give to leading order, $\delta A_{1}=\left(e g_{\pi N} / 32 F_{\pi}^{2}\right) \mu$ due to the presence of a logarithmic singularity and consequently contributes at next-to-leading order in the quark mass expansion of $E_{0+}$. Thus this novel term originates from the presence of Goldstone bosons which leads to the existence of non analytical terms $(\mu \sim \sqrt{\hat{m}})$ invalidating the assumptions $\left(\delta A_{1}=\mathcal{O}\left(\mu^{2}\right)\right)$ used to derive what one should not call a "LET" but a "LEG" (low energy guess as defined by G. Ecker, this workshop). Clearly, the expansion in $\mu$ is slowly converging, the coefficient of the term of order $\mu^{2}$ is so large that it compensates the leading term proportional to $\mu$. Therefore, for a meaningful prediction one has to go further in the expansion. To order $\mu^{3}$ one gets -1.14 using the conventional units of $10^{-3} / M_{\pi^{+}}$to be compared with -3.35 at leading order and 0.89 at next-to-leading order. These results show that the LET for $E_{0+}$ is practically useless and that it turns out to be very hard to determine this quantity theoretically, making it not the best test of chiral dynamics.

However, it is very easy to derive LETs for the $P$-waves within CHPT. These have never been looked at before. They take the following forms:

$$
\begin{aligned}
& \frac{1}{|\vec{q}|} P_{1}=-\frac{e g_{\pi N}}{8 \pi m^{2}}\left\{1+\kappa_{p}+\left[-1-\frac{\kappa_{p}}{2}+\frac{g_{\pi N}^{2}}{48 \pi}(10-3 \pi)\right] \mu+\mathcal{O}\left(\mu^{2}\right)\right\}, \\
& \frac{1}{|\vec{q}|} P_{2}=-\frac{e g_{\pi N}}{8 \pi m^{2}}\left\{-1-\kappa_{p}+\left[3+\kappa_{p}-\frac{g_{\pi N}^{2}}{12 \pi}\right] \frac{\mu}{2}+\mathcal{O}\left(\mu^{2}\right)\right\} .
\end{aligned}
$$


They are very fastly converging functions of $\mu$. Indeed the terms of $\mathcal{O}(\mu)$ contributes for $6 \%$ (less than $0.1 \%$ ) to $P_{1}\left(P_{2}\right)$ respectively. They thus constitute a very good test of chiral dynamics. Writing them in the conventional units $|\vec{q}||\vec{k}| 10^{-3} / M_{\pi^{+}}$, one gets 10.3 and -10.9 for $P_{1}$ and $P_{2}$, respectively. Notice that already at next to leading order $P_{1}$ and $P_{2}$ differ. Consequently, the value $E_{1+} \equiv 0$ is excluded. I will end the discussion on the $P$-waves here just emphasizing that they depend weakly on energy, they show no cusp effect and they have a small imaginary part.

\subsection{Results and discussion}

All the CHPT results presented in this section are preliminary. Let me first concentrate on the real part of $E_{0+}$. The reanalysis of the data agree on its value at threshold, $E_{0+} \sim-2.10^{-3} / M_{\pi^{+}}$(accidentally the experimental result and the LEG are in agreement) but disagree on its energy dependence, the difference between the

Fig.2 Real part of $E_{0+}$.

reanalysis coming essentially from different assumptions on the $P$-waves. We just saw that $P_{1}$ and $P_{2}$ are constrained since they obey LETs, these were however not known at the time of the reanalysis. Bergstrom [13] and Tiator [13] show a rather strong energy dependence while Bernstein [13] has a weak one. At present this weak energy dependence seems to be ruled out by the LETs which are violated in Bernstein's reanalysis. In fig. 2 is shown the CHPT result. There, some isospin breaking effects have been taken into account by differentiating in the loops the charge pion mass from the neutral one. The two curves represent the two different ways of estimating the low energy constants discussed previously. The full curve corresponds to the best fit to the differential and total cross-sections, the band corresponding to a \pm 1 standard deviation. The dotted curve corresponds to the determination via resonance exchange 
where the values of the off-shell parameters of the $\Delta \gamma N$ vertex and the $\Delta$ propagator are again obtained by a best fit to the data constrained so that these values lie within the error bars of the theoretical estimates. One notes that the value of $d_{7}$ is somewhat independent of the method used. The value of $E_{0+}$ at threshold lies between -1 to -1.5 somewhat smaller in magnitude than the experimental one. It is very much constrained by the bell shape type of the angular distribution of the differential cross-sections for a photon energy larger than $150 \mathrm{MeV}$ as seen in Fig.3 which shows how good the fits are. The energy dependence is weaker than in the reanalysis of Bergstrom and Tiator, their value of $\operatorname{Re} E_{0+}$ at the $\pi^{+} n$ threshold being close to zero to be compared with the CHPT one of -0.5 . Thus the difference of -2 . between the two threshold cannot be obtained within a $\mathcal{O}\left(q^{4}\right)$ calculation. We had already seen that the $\mu$ expansion of $E_{0+}$ is very slowly converging, this of course demands a two-loop calculation to test the validity of the results presented here. Of course more precise measurements are needed too.

Fig.3 Angular distribution of $d \sigma / d \Omega: E_{\gamma}=151.4$ (left), $=153.7 \mathrm{MeV}$ (right).

The imaginary part of $E_{0+}$ is a very important quantity to look at and this for the following reasons. First, its result is genuine since no counterterm is involved in its calculation. Second, it is related to the change of its real part through a dispersion relation. This can also be seen very easily by using a simple but however realistic model where $E_{0+}=a-b \sqrt{1-E_{\gamma} / E_{\gamma}^{(+)}}$with $E_{\gamma}^{(+)}$the photon energy at the $\pi^{+} n$ threshold (a good fit is obtained with $a=0.3$ and $b=5.7$ in the units $10^{-3} / M_{\pi^{+}}$). Another point has been stressed by Bernstein [16]. The imaginary part of $E_{0+}$ is related to the $\pi N$ scattering lengths through the Fermi Watson theorem. Thus a measure of the imaginary part would lead to another way of determining these important quantities. Within CHPT to $\mathcal{O}\left(q^{4}\right), \operatorname{Im} E_{0+}$ stays below $10^{-3} / M_{\pi^{+}}$for $E_{\gamma}<160 \mathrm{MeV}$. It is too small, as we have already seen, to account for the strong energy dependence of $\operatorname{Re} E_{0+}$. For all these reasons it would be very interesting to have a measure of $\operatorname{Im} E_{0+}$. This can be done through a measurement of polarized observables. Two of them turn out to be particularly sensitive to this quantity, these are the polarized target asymmetry $\mathrm{T}$ and the recoil polarization $\mathrm{P}$ as can easily be seen by looking at their definitions:

$$
\begin{aligned}
& T \propto \operatorname{Im}\left(\left(E_{0+}+\cos \theta P_{1}\right)\left(P_{2}-P_{3}\right)\right. \\
& P \propto-\operatorname{Im}\left(\left(E_{0+}+\cos \theta P_{1}\right)\left(P_{2}+P_{3}\right)\right.
\end{aligned}
$$


The CHPT results are shown in Fig. 4. One sees that though $\mathrm{P}$ stays very small, $\mathrm{T}$ reaches 15 to $20 \%$ over a wide range of angles. This makes this observable a good candidate for a measurement of $\operatorname{Im} E_{0+}$.

Fig.4 Polarized observables $P$ and $T$ for $E_{\gamma}=151.4 \mathrm{MeV}$.

\section{Threshold Pion Electroproduction}

Fig.5 Angular distribution of $d \sigma_{T} / d \Omega$ for $k^{2}=-0.1 \mathrm{GeV}^{-2}$.

I will now discuss some rather new data on electroproduction. Welch et al.[2] have published the $S$-wave cross section for the reaction $\gamma^{\star} p \rightarrow \pi^{0} p$ very close to the photon point. This measurement is a quantum step compared to previous determinations which mostly date back to the seventies when pion electroproduction was still a hot 
topic in particle physics. In this experiment, $k^{2}$ varied between -0.04 and $-0.10 \mathrm{GeV}^{-2}$ and the $S$-wave cross section could be extracted with an unprecedented accuracy (see fig.2 in [2]). This is also the kinematical regime where a CHPT calculation might offer some insight. Indeed, in ref.[17] it was shown that the $k^{2}$-dependence of this cross section seems to indicate the necessity of loop effects. With conventional models including e.g. form factors and the anomalous magnetic moment coupling the trend of the data can not be described. However, the corrections from the one loop diagrams to the tree level prediction are substantial. This gives further credit to the previously made statement that a calculation beyond next-to-leading order should be performed. Very recently, Distler et al. [3] have measured the angular distribution of the differential transverse cross section at $k^{2}=-0.1 \mathrm{GeV}^{-2}$. Even though this value is somewhat too high for a good test of CHPT ( there, the one-loop order corrections are of the order of 50\%) it is interesting to see that the preliminary experimental results show the same trends as the CHPT ones and are at variance with the pseudo-vector Born approximation (see Fig.5). It would be of particular interest to have experimental informations at $k^{2}=-0.05 \mathrm{GeV}^{-2}$.

The last topic I want to address in this section concerns the determination of the nucleon axial radius from charged pion electroproduction. Let me briefly explain how the axial form factor comes into play. The basic matrix element to be considered is the time-ordered product of the electromagnetic (vector) current with the interpolating pion field sandwiched between nucleon states. Now one can use the PCAC relation and express the pion field in terms of the divergence of the axial current. Thus, a commutator of the form $[\mathrm{V}, \mathrm{A}]$ arises. Current algebra tells us that this gives an axial current between the incoming and outgoing nucleon fields and, alas, the axial form factor. The isospin factors combine in a way that they form a totally antisymmetric combination which can not be probed in neutral pion production. These ideas were formalized in the venerable low-energy theorem (LET) due to Nambu, Lurie and Shrauner [18] for the isospin-odd electric dipole amplitude $E_{0+}^{(-)}$in the chiral limit,

$$
E_{0+}^{(-)}\left(M_{\pi}=0, k^{2}\right)=\frac{e g_{A}}{8 \pi F_{\pi}}\left\{1+\frac{k^{2}}{6} r_{A}^{2}+\frac{k^{2}}{4 m^{2}}\left(\kappa_{V}+\frac{1}{2}\right)+\mathcal{O}\left(k^{3}\right)\right\}
$$

Therefore, measuring the reactions $\gamma^{\star} p \rightarrow \pi^{+} n$ and $\gamma^{\star} n \rightarrow \pi^{-} p$ allows to extract $E_{0+}^{(-)}$ and one can determine the axial radius of the nucleon, $r_{A}$. This quantity measures the distribution of spin and isospin in the nucleon, i.e. probes the Gamov-Teller operator $\sigma \cdot \tau$. A priori, the axial radius is expected to be different from the typical electromagnetic size, $r_{\mathrm{em}} \simeq 0.85 \mathrm{fm}$. It is customary to parametrize the axial form factor $G_{A}\left(k^{2}\right)$ by a dipole form, $G_{A}\left(k^{2}\right) / g_{A}=\left(1-k^{2} / M_{A}^{2}\right)^{-2}$ which leads to the relation $r_{A}=\sqrt{12} / M_{A}$. The axial radius determined from electroproduction data is typically $r_{A}=0.59 \pm 0.04 \mathrm{fm}$ whereas (anti)neutrino-nucleon reactions lead to somewhat larger values, $r_{A}=0.65 \pm 0.03 \mathrm{fm}$. This discrepancy is usually not taken seriously since the values overlap within the error bars. However, it was shown in ref.[19] that pion loops modify the LET (2.15) at order $k^{2}$ for finite pion mass. In the heavy mass formalism, the coefficient of the $k^{2}$ term reads

$$
\frac{1}{6} r_{A}^{2}+\frac{1}{4 m^{2}}\left(\kappa_{V}+\frac{1}{2}\right)+\frac{1}{128 F_{\pi}^{2}}\left(1-\frac{12}{\pi^{2}}\right)
$$


where the last term in (2.16) is the new one. This means that previously one had extracted a modified radius, the correction being $3\left(1-12 / \pi^{2}\right) / 64 F_{\pi}^{2} \simeq-0.046 \mathrm{fm}^{2}$. This closes the gap between the values of $r_{A}$ extracted from electroproduction and neutrino data. It remains to be seen how the $1 / m$ suppressed terms will modify the result (2.16). Such investigations are underway.

\section{Threshold Two Pion Photoproduction:}

At threshold in the center-of-mass frame (i.e. $\vec{q}_{1}=\vec{q}_{2}=0$ ), the two-pion photoproduction current matrix element can be decomposed into amplitudes as follows if we work to first order in the electromagnetic coupling $e$,

$$
T \cdot \epsilon=\chi_{f}^{\dagger}\left\{i \vec{\sigma} \cdot(\vec{\epsilon} \times \vec{k})\left[M_{1} \delta^{a b}+M_{2} \delta^{a b} \tau^{3}+M_{3}\left(\delta^{a 3} \tau^{b}+\delta^{b 3} \tau^{a}\right)\right]\right\} \chi_{i}
$$

with $\chi_{i, f}$ two-component Pauli-spinors and isospinors and we used the gauge $\epsilon_{0}=0$. To leading order in the chiral expansion, only the amplitudes $M_{2}$ and $M_{3}$ are non vanishing, with $M_{2}=-2 M_{3}$ [20]. Therefore the production of two neutral pions is strictly suppressed. One can derive LETs for $M_{2,3}$. They read:

$$
M_{2}=-2 M_{3}=\frac{e}{4 m F_{\pi}^{2}}\left(2 g_{A}^{2}-1-\kappa_{V}\right)
$$

There exists in the literature some incomplete determination of these LETs [21]. The authors did not use the most general effective Lagrangian and thus did not get the term proportional to $\kappa_{V}$. At next order in the chiral expansion, one has to consider kinematical $(1 / m)$ corrections, one-loop contributions and tree graphs with insertion of the $\Delta(1232)$ (chirally expanded). Note that, to this order, the $\Delta$ contributions are absent in the $\pi^{0} \pi^{0}$ channel. Here we use the principle of resonance saturation to estimate the counterterms. At this order one has a LET for $M_{1}$ :

$$
M_{1}=\frac{e g_{A}^{2} M_{\pi}}{4 m F_{\pi}^{2}}
$$

and the $M_{2,3}$ have a somewhat more complicated expressions (see ref.[20]). Their loop contributions have a non-zero imaginary part even at threshold which comes from the rescattering graph. Due to unitarity the pertinent loop functions have a right hand cut starting at $s=\left(m+M_{\pi}\right)^{2}$ (the single pion production threshold) and these functions are here to be evaluated at $s=\left(m+2 M_{\pi}\right)^{2}$ (the two- pion production threshold).In Fig. 6 are shown the total cross sections for $\gamma p \rightarrow \pi^{+} \pi^{-} p, \pi^{+} \pi^{0} n$ and $\pi^{0} \pi^{0} p$. Here the calculation was done with the correct phase-space and approximating the amplitudes in the threshold region through their threshold values in the case of the two last reactions. For the first one the first correction above threshold is also shown [20]. At $E_{\gamma}=320 \mathrm{MeV}$, the total cross section for $\pi^{0} \pi^{0}$ production is $0.5 \mathrm{nb}$ whereas the competing $\pi^{+} \pi^{0} n$ final state has $\sigma_{\text {tot }}=0.07 \mathrm{nb}$. Double neutral pion production reaches $\sigma_{\text {tot }}=1.0 \mathrm{nb}$ at $E_{\gamma}=324.3 \mathrm{MeV}$ in comparison to $\sigma_{\text {tot }}\left(\gamma p \rightarrow \pi^{0} \pi^{+} n\right)=0.26$ $\mathrm{nb}$ and $\sigma_{\text {tot }}\left(\gamma p \rightarrow \pi^{+} \pi^{-} p\right)<0.1 \mathrm{nb}$. This means that for the first $10 \ldots 12 \mathrm{MeV}$ above $\pi^{0} \pi^{0}$ threshold (chiral window), one has a fairly clean signal and much more neutrals than expected. Of course, the above threshold correction for all the channels should be 
calculated systematically. However the first correction, which vanishes proportional to $\left|\vec{q}_{i}\right|(\mathrm{i}=1,2)$ at threshold, has been calculated and found to be small. It is therefore conceivable that the qualitative features described above will not change if even higher corrections are taken into account. We hope to report on these in the not too distant future. From the experimental side TAPS seems to have seen some $\pi^{0}$ 's close to threshold. For more detail, see T. H. Walcher, these proceedings.

Fig.6 Total cross sections (in nb) for the $\gamma p$ initial state

\section{Summary and Outlook}

Pion photo- and electroproduction in the threshold region is a good testing ground of the chiral nucleon structure. A one-loop CHPT calculation gives a satisfactory description of most of the existing observables. The pertinent results of our investigation can be summarized as follows:

- The LET for $E_{0+}$ is practically useless. At variance with what is usually believed the $P$-waves are the quantities providing a good test of chiral dynamics.

- We have stressed the importance of the imaginary part of $E_{0+}$.

- We have obtained a better understanding of the axial radius of the nucleon as measured in charged electroproduction.

- We have seen that there is a window of about $10 \mathrm{MeV}$ above $\pi^{0} \pi^{0}$ threshold in which one should detect much more neutrals than expected in two pion photoproduction.

At this stage some more efforts are needed on the theoretical side to pin down the isospin breaking effects. Also a two loop calculation is mandatory to determine the validity of the results presented here, especially in the case of the S-wave observables. On the experimental side, it is clear that in order to test CHPT, one has to measure close to threshold and to get to very accurate data. I want to stress again that it would be very important to have a measure of the imaginary part of $E_{0+}$ as well as a good determination of the energy dependence of its real part. 


\section{Acknowledgements}

I would like to thank the organizers for their invitation and kind hospitality.

\section{References}

[1] E. Mazzucato et al., Phys. Rev. Lett. 57 (1986) 3144; R. Beck et al., Phys. Rev. Lett. 65 (1990) 1841

[2] T. P. Welch et al., Phys. Rev. Lett. 69 (1992) 2761

[3] T. H. Walcher, these proceedings

[4] H. Leutwyler, see for exemple these proceedings and "Principles of Chiral Perturbation Theory", lectures given at the Workshop "Hadrons 1994", Gramado, Brasil, BUTP-94/13

[5] Ulf-G. Meißner, Rep. Prog. Phys. 56 (1993) 903

[6] V. Bernard, N. Kaiser and Ulf-G. Meißner, Z. Phys. A348 (1994) 317; in preparation

[7] V. Bernard, N. Kaiser and Ulf-G. Meißner, Nucl. Phys. B383 (1992) 442

[8] J. Gasser, M.E. Sainio and A. Švarc, Nucl. Phys. B307 (1988) 779

[9] G. Ecker, "Chiral Invariant Renormalization of the Pion-Nucleon Interaction", Phys. Lett. B, in print, UWThPh-1994-1

[10] G. Ecker, J. Gasser, A. Pich and E. de Rafael, Nucl. Phys. B321 (1989) 311; G. Ecker, J. Gasser, H. Leutwyler, A. Pich and E. de Rafael, Phys. Lett. B223 (1989) 425

[11] A. I. Vainshtein and V. I. Zakharov, Nucl. Phys. B36 (1972) 589; P. de Baenst, Nucl. Phys. B24 (1970) 633

[12] V. Bernard, N. Kaiser, J. Gasser and Ulf-G. Meißner, Phys. Lett. B268 (1991) 291 and references therein

[13] A. M. Bernstein and B. R. Holstein, Comments Nucl. Par. Phys. 20 (1991) 197; J. Bergstrom, Phys. Rev. C44 (1991) 1768; L. Tiator, "Meson Photo- and Electroproduction", lecture given at the II TAPS Workshop, Alicante, 1993

[14] R. Davidson and N. C. Mukhopadhyay, Phys. Rev. Lett. 60 (1988) 748; E. Mazzucato et al., Phys. Rev. Lett. 60 (1988) 749; S. Nozawa, T.-S.H. Lee and B. Blankleider, Phys. Rev. C41 (1990) 213; A. N. Kamal, Phys. Rev. Lett. 63 (1989) 213

[15] S. Weinberg, Physica A96 (1979) 327

[16] A. M. Bernstein, $\pi N$ Newsletter 9 (1993) 55

[17] V. Bernard, N. Kaiser, T.-S.H. Lee and Ulf-G. Meißner, Phys. Rev. Lett. 70 (1993) 387; Phys. Reports, in print

[18] Y. Nambu and D. Lurié, Phys. Rev. 125 (1962) 1429; Y. Nambu and E. Shrauner, Phys. Rev. 128 (1962) 862

[19] V. Bernard, N. Kaiser and Ulf-G. Meißner, Phys. Rev. Lett. 69 (1992) 1877

[20] V. Bernard, N. Kaiser and Ulf-G. Meißner, "Threshold two-pion photo- and electroproduction: More neutrals than expected", CRN-94/14, Nucl. Phys. A, in print

[21] R. Dahm and D. Drechsel, in Proc. Seventh Amsterdam Mini-Conference, eds. H. P. Blok, J. H. Koch and H. De Vries, Amsterdam, 1991; M. Benmerrouche and E. Tomusiak, Phys. Rev. Lett. 73 (1994) 400

This article was processed by the author using the $\mathrm{TEX}_{\mathrm{E}}$ macro package from Springer-Verlag. 
This figure "fig1-1.png" is available in "png" format from: http://arxiv.org/ps/hep-ph/9408323v1 
This figure "fig1-2.png" is available in "png" format from: http://arxiv.org/ps/hep-ph/9408323v1 